\title{
Papers
}

\section{Is it possible to exclude a diagnosis of myocardial damage within six hours of admission to an emergency department? Diagnostic cohort study Topic: 15}

\author{
K R Herren, K Mackway-Jones, C R Richards, C J Seneviratne, M W France, L Cotter
}

\begin{abstract}
Objective To assess the clinical efficacy and accuracy of an emergency department based six hour rule-out protocol for myocardial damage.

Design Diagnostic cohort study.

Setting Emergency department of an inner city university hospital.

Participants 383 consecutive patients aged over 25 years with chest pain of less than 12 hours' duration who were at low to moderate risk of acute myocardial infarction.

Intervention Serial measurements of creatine kinase $\mathrm{MB}$ mass and continuous ST segment monitoring for six hours with 12 leads.

Main outcome measure Performance of the diagnostic test against a gold standard consisting of either a 48 hour measurement of troponin $\mathrm{T}$ concentration or screening for myocardial infarction according to the World Health Organization's criteria. Results Outcome of the gold standard test was available for 292 patients. On the diagnostic test for the protocol, 53 patients had positive results and 239 patients had negative results. There were 18 false positive results and one false negative result. Sensitivity was $97.2 \%$ (95\% confidence interval $95.0 \%$ to $99.0 \%)$, specificity $93.0 \%$ (90.0\% to $96.0 \%)$, the negative predictive value $99.6 \%$, and the positive predictive value $66.0 \%$. The positive likelihood ratio was 13.9 and the negative likelihood ratio 0.03 . Conclusions The six hour rule-out protocol for myocardial infarction is accurate and efficacious. It can be used in patients presenting to emergency departments with chest pain indicating a low to moderate risk of myocardial infarction.
\end{abstract}

\section{Introduction}

Chest pain accounts for 2-4\% of all new presentations at emergency departments in the United Kingdom. ${ }^{12}$ Current best practice requires that all patients with a possible cardiac problem be admitted for at least 12-24 hours for further tests. ${ }^{34}$ In the United Kingdom 30\% of such patients are admitted and $70 \%$ discharged, whereas in the United States around 60\% are admitted..$^{15}$
Acute myocardial infarctions are missed in about $3.5 \%$ of patients admitted to emergency departments in the United States, and such patients are subsequently discharged. In the United Kingdom recent evidence suggests that around $6 \%$ of patients discharged from emergency departments may have prognostically important myocardial damage. ${ }^{6}$ Although many interventions, including drugs and surgery, can reduce mortality, patients benefit only if correctly identified. ${ }^{5} 78$ Mortality in patients who are discharged with missed acute myocardial infarctions is four times greater than in those who are admitted to hospital. ${ }^{9}$

Diagnostic strategies applied to patients with possible cardiac chest pain in emergency departments have two aims: firstly, the prompt identification of patients with acute myocardial infarction allowing early initiation of time dependent interventions, and, secondly, the exclusion of myocardial damage in a timely and clinically accurate manner so that patients can be discharged appropriately. The first of these is a rule-in requirement (rule in myocardial infarction), and the second a rule-out requirement (rule out myocardial infarction). Failure of the rule-out requirement results in inappropriate discharge of patients with acute myocardial infarction.

Sensitivity and specificity are measures of the clinical efficacy of a diagnostic test, whereas clinical accuracy is determined by using predictive values. A test needs to be highly sensitive to be useful in a rule-out protocol but highly specific in a rule-in protocol. Although sensitivity and specificity are less susceptible to disease prevalence than measures of clinical accuracy, particular values apply only to populations with a similar spectrum of disease to that in which they were derived. Furthermore, failure to follow up patients with negative test results (verification bias) may lead to seriously misleading results. ${ }^{10}$

Traditionally, ruling out myocardial infarction is accomplished by combining serial measurements of cardiac enzymes (creatine kinase, aspartate transaminase, and lactate dehydrogenase) with serial electrocardiograms. The sensitivity is $96.2 \%$ in patients with chest pain 24 hours after arrival at an emergency department. $^{3}$ Although accurate, this approach requires admission for a minimum of 24 hours and is therefore neither cost effective nor timely. Ideally one

\author{
Department of \\ Emergency \\ Medicine, \\ Manchester Royal \\ Infirmary, \\ Manchester \\ M13 9WL \\ K R Herren \\ research fellow \\ K Mackway-Jones \\ consultant \\ C R Richards \\ research assistant \\ Department of \\ Clinical \\ Biochemistry, \\ Manchester Royal \\ Infirmary \\ C J Seneviratne \\ consultant clinical \\ scientist \\ M W France \\ consultant \\ Manchester Heart \\ Centre, Manchester \\ Royal Infirmary \\ L Cotter \\ consultant \\ Correspondence to: \\ K Mackway-Jones \\ kevin.mackway- \\ jones@man.ac.uk
}

BMJ 2001;323:1-4 
test would both rule out and rule in myocardial infarction. No single test is currently sensitive and specific enough to rule out myocardial infarction in the first 6-8 hours after onset of chest pain, ${ }^{11}$ and electrocardiography, although highly specific (77-100\%), has too low a sensitivity $(56 \%)$ to be used in this way. ${ }^{12}{ }^{13}$

In the United States, chest pain assessment units are increasingly being established in emergency departments. ${ }^{1-16}$ These combine serial measurements of creatine kinase MB with continuous ST segment monitoring to rule out myocardial infarction. Although this approach is safe, ${ }^{7}$ no study has assessed its clinical efficacy with a gold standard for both admitted and discharged patients. We aimed to assess the clinical efficacy and accuracy of an emergency department based six hour rule-out protocol for myocardial infarction.

\section{Patients and methods}

Consecutive patients presenting with chest pain to Manchester Royal Infirmary's emergency department between 20 October 1997 and 30 October 1998 were eligible for our study. We included patients if they were aged over 25, the chest pain had lasted less than 12 hours, there was no history of trauma, and no other medical cause of chest pain had been diagnosed. We excluded patients if they had electrocardiographic evidence of acute myocardial infarction or myocardial ischaemia, were hypotensive, had an arrhythmia, or required admission for another medical or social reason. We also excluded patients if the protocol was not followed or they did not consent to participate. Ethical approval for our study was provided by the Central Manchester local research ethics committee (CM/97/137) and all patients gave informed consent.

All the patients underwent 12 lead electrocardiography before admission to the unit for assessment of their chest pain. They were given aspirin $300 \mathrm{mg}$ (unless an absolute contraindication existed), and an intravenous cannula was placed.

The diagnostic test series consisted of serial measurements of creatine kinase $\mathrm{MB}$ mass and continuous ST segment monitoring for six hours with 12 leads. If chest pain had lasted less than three hours we measured creatine kinase MB mass three hours after onset, six hours after onset, and after six hours of monitoring. If chest pain had lasted 3-12 hours we measured creatine kinase MB mass on arrival and three hours later.

Creatine kinase MB mass was measured with a specific immunoassay (Elecsys 2010 analyser, Roche Diagnostics, Lewes, Sussex). A positive test result was an absolute concentration greater than $5 \mu \mathrm{g} / \mathrm{l}$ or an increase of $3 \mu \mathrm{g} / \mathrm{l}$ between consecutive samples.

The ST segments were continuously monitored with the appropriate module in a Medical PC Scout monitor (Spacelabs, Wokingham, Kent). The alarm on the monitor was set to go off after a change in ST segment elevation or depression of greater than $2 \mathrm{~mm}$ in any one single lead or $1 \mathrm{~mm}$ in any three leads. The printout was interpreted by the clinician responsible for the patient. At the end of the monitoring period a 12 lead electrocardiogram was produced.

The test result was positive if there was a positive result for creatine kinase $\mathrm{MB}$ mass or if an important change occurred in the ST segment. The test result was negative if there was a negative result for creatine kinase MB mass and no important change occurred in the ST segment.

Patients were admitted to hospital under the care of the acute medical team if they were had positive results on the test or developed another medical or social reason for admission. All other patients were discharged to the care of their general practitioners with an advice card that explained the result and outlined reasons for seeking further care. All these patients were advised to see their general practitioners at the first possible opportunity.

Patients were asked to return after two days for measurement of troponin $\mathrm{T}$ concentrations. If they failed to do so they were contacted by telephone and asked to return for the test. Inpatients either underwent measurement of troponin $\mathrm{T}$ concentration 48 hours after admission or were screened for myocardial infarction according to the World Health Organization's criteria. ${ }^{17}$ Troponin $\mathrm{T}$ was measured by a second generation specific immunoassay (Elecsys 2010 analyser, Roche Diagnostics). A concentration of greater than $0.1 \mu \mathrm{g} / \mathrm{l}$ indicated myocardial damage.

All patients were asked to return to the clinic after one month, where a further history was taken and an examination and 12 lead electrocardiography performed. We contacted the general practitioners of patients who did not attend. Sensitivity, specificity, positive and negative predictive values, and likelihood ratios were calculated with standard formulas. ${ }^{18}$

\section{Results}

Overall, 383 patients who attended with chest pain underwent the full protocol (figure). Fourteen were excluded for violation of the protocol: three did not complete the test and 11 fulfilled an exclusion criteria. One patient withdrew. Of the 368 patients who completed the diagnostic test, 254 had had chest pain for more than three hours and 114 for less than three hours.

Eleven patients withdrew from follow up and 65 had no gold standard measure of outcome- one patient who had been admitted to hospital had insufficient results to confirm the outcome, 63 discharged patients did not return for measurement of troponin $\mathrm{T}$ concentration, and one patient had the wrong sample taken. These patients had the same risk profile for myocardial infarc-

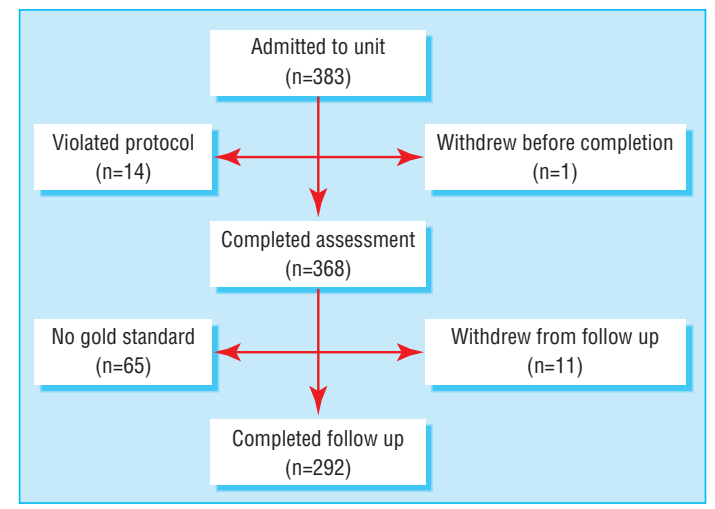

Trial profile 
tion and the same sex ratio as the patients with a gold standard. They were more likely to be aged less than 40 years. Sixty one of the 65 patients were traced four weeks or more after discharge. All were still alive, and none had had a myocardial infarction.

The outcome of the gold standard test was available for 292 patients: 288 were appropriately timed for measurement of troponin $\mathrm{T}$ concentration and four underwent serial electrocardiography and serial measurements of cardiac enzymes.

Fifty three patients were protocol positive: in 40 the first measurement of creatine kinase MB mass was raised, in five the second measurement was raised, in one the third measurement was raised, and one had an increase greater than $3 \mu \mathrm{g} / \mathrm{l}$ between consecutive samples. Six were protocol positive on ST segment monitoring. In total, 239 patients were protocol negative.

Overall, 238 patients were also gold standard negative and one was gold standard positive (table). The false negative patient had a troponin $\mathrm{T}$ concentration of $0.11 \mu \mathrm{g} / \mathrm{l}$ at two days. On review at one month she was well, with no electrocardiographic evidence of myocardial damage.

Sensitivity of the diagnostic test was 97.2\% (95\% confidence interval $95.0 \%$ to $99.0 \%$ ), specificity $93.0 \%$ $(90.0 \%$ to $96.0 \%)$; the negative predictive value was $99.6 \%$ and the positive predictive value $66.0 \%$. The positive likelihood ratio was 13.9 and the negative likelihood ratio 0.03 .

\section{Discussion}

Chest pain assessment units have been established in the United States to manage the problem of accurately ruling out myocardial damage in unselected patients with chest pain of a cardiac type. Evidence suggests that this approach is safe, ${ }^{74-16}$ but the diagnostic test series used have never been evaluated against a gold standard, and the possibility of verification bias remains. Furthermore, the spectrum of disease and characteristics of a population are important in assessing a new diagnostic test. ${ }^{10}$ Our study is the first to report both the diagnostic efficacy and accuracy of assessing chest pain in such a unit and its use in a UK population.

A troponin T concentration greater than $0.1 \mu \mathrm{g} / \mathrm{l}$ at two days was used as a gold standard marker for myocardial damage. Several studies have shown that an increase of the troponin $\mathrm{T}$ concentration to this level is a strong independent marker of risk in patients with chest pain. ${ }^{19}{ }^{20}$ Although a higher cut-off concentration could have been chosen to define acute myocardial infarction, the lower level is more appropriate for use in this study of a rule-out protocol, where sensitivity is paramount.

The six hour diagnostic protocol effectively ruled out myocardial damage with sufficient clinical efficacy to allow both rapid identification and safe discharge. The patient who was negative for the test and positive for the gold standard had a troponin T concentration just above the upper limit of normal. Later analysis of samples from this patient showed no increase in creatine kinase concentration, creatine kinase MB mass, or creatine kinase isoforms at any time and no increase in troponin $\mathrm{T}$ concentration in any of the protocol
Number of patients categorised as positive or negative by rule-out protocol (serial measurement of creatine kinase MB mass and continuous monitoring of ST segment changes) and by gold standard (troponin T concentration at 48 hours or serial electrocardiography and cardiac enzymes over 24 hours)

\begin{tabular}{lccc} 
Result in chest pain assessment & \multicolumn{2}{c}{ Gold standard } & \\
\cline { 2 - 3 } unit & Positive & Negative & Total \\
\hline Positive & 35 & 18 & 53 \\
\hline Negative & 1 & 238 & 239 \\
\hline Total & 36 & 256 & 292 \\
\hline
\end{tabular}

samples. No evidence suggested that this person had an acute myocardial infarction.

Although ST segment monitoring is used in many coronary and intensive care units and during anaesthesia, ${ }^{21} 22$ its utility for diagnosing chest pain has not been evaluated in an emergency department. Of the six patients with changes in their ST segment during the protocol, two had evolving myocardial infarctions and both subsequently received thrombolysis after confirmatory 12 lead electrocardiograms. No other patients received thrombolysis in the first 12 hours after arrival. Thus monitoring effectively detected important evolving changes in the ST segment.

Because patients left the protocol at the first positive test result it was not possible to calculate the actual performance of individual tests. Although it is likely that the two evolving myocardial infarctions that were identified by ST segment monitoring would have been picked up later by increases in creatine kinase $\mathrm{MB}$ mass, this cannot be proved. It is certain, however, that the inclusion of ST segment monitoring in the protocol allowed the earliest possible identification of the two evolving acute myocardial infarctions and therefore expedited thrombolysis, a time dependent intervention.

Over $80 \%$ of available patients had a gold standard done in this study. The populations served by an inner city emergency department such as the Manchester Royal Infirmary are highly mobile and diverse; many of the patients who did not return were homeless, non-local, or migrant. Although $100 \%$ follow up is desirable, this test was evaluated in a real life population, and exclusion of patients who were not

\section{What is already known on this topic}

In emergency departments in the United Kingdom only $30 \%$ of patients with chest pain indicating a low to moderate risk of myocardial infarction are admitted to hospital

Some $6 \%$ of those discharged have undiagnosed myocardial damage

\section{What this study adds}

An emergency department based chest pain assessment unit protocol to rule out myocardial damage is sensitive enough to allow safe discharge of patients at low to moderate risk of myocardial infarction within six hours

Such units can also reduce the number of patients admitted unnecessarily 
local or those who were homeless would have introduced bias by affecting the spectrum of disease.

The six hour rule-out protocol for myocardial infarction is both clinically accurate and efficacious in patients in the emergency department with chest pain indicating a low to moderate risk of myocardial infarction. The protocol reduces unnecessary admissions and inappropriate discharges and can also facilitate early thrombolysis in some patients who might otherwise not receive it.

Funding: This study was supported by a grant from the North West office of the NHS Executive (RDO/21/11).

Contributors: KRH was responsible for the detailed study design, oversaw the running of the assessment unit, and participated in data collection and analysis and writing the paper. KM-J had the original idea and initiated the study, oversaw the study design and data collection, and participated in data analysis and the writing of the paper; he will act as guarantor. CRR helped with the study design, undertook data collection, and participated in the writing of the paper. CJS, MWF, and LC participated in the study design, data interpretation, and writing of the paper.

Competing interests: None declared.

1 Fothergill NJ, Hunt MT, Touquet R. Audit of patients with chest pain presenting to an accident and emergency department over a 6-month period. Arch Emerg Med 1993;10:155-60.

2 Emerson PA, Russell NJ, Wyatt J, Crichton N, Pantin CFA, Morgan AD, et al. An audit of doctors management of patients with chest pain in the accident and emergency department. QJ Med 1989;70:213-20.

3 Lee TH, Rouan GW, Weisberg M, Brand DA, Cook EF, Acampora D, et al. Sensitivity of routine clinical criteria for diagnosing myocardial infarction within 24 hours of hospitalisation. Ann Intern Med 1987;106:181-6.

4 Mair J, Artner-Dworzak E, Lechleitner P, Smidt J, Wagner I, Dienstl F, et al. Cardiac troponin $\mathrm{T}$ in diagnosis of acute myocardial infarction. Clin Chem $1991 ; 37: 845-52$

5 ISIS-2 (Second international study of infarct survival) collaborative group. ISIS-2: a randomised comparison of streptokinase vs tissue plasminogen activator vs anistreplase and of aspirin plus heparin vs aspi-
rin alone in 41299 cases of suspected acute myocardial infarction. Lancet 1992:339:753-70

6 Collinson PO, Premachandram S, Hashemi K. Prospective audit of incidence of prognostically important myocardial damage in patients discharged from emergency department. BMJ 2000;320:1702-5.
7 Farkouh ME, Smars PA, Reeder GS, Zinsmeister AR, Evans RW, Meloy TD, et al. A clinical trial of a chest pain observation unit for patients with unstable angina. N Engl J Med 1998;339:1882-8.

8 King III SB, Lembo NJ, Weintraub WS, Kosinski AS, Barnhart HX, Kutner $\mathrm{MH}$, et al. A randomised controlled trial comparing coronary angioplasty with coronary bypass surgery. N Engl J Med 1994;331:1044-50.

9 Lee TH, Rouan GW, Weisberg M, Brand DA, Acampora D, Stasiulewicz C, et al. Clinical characteristics and natural history of patients with acute myocardial infarction sent home from the emergency department. Am J Cardiol 1987;60:219-24

10 Ransohoff DF, Feinstein AR. Problems of spectrum and bias in evaluating the efficacy of diagnostic tests. N Engl J Med 1978;299:926-30.

11 Bakker AJ, Koelemay MJW, Gorgels PMC, Van Vlies B, Smits R., Tijssen $\mathrm{JG}$, et al. Failure of new biochemical markers to exclude acute myocardial infarction at admission. Lancet 1993:342:1220-2.

12 Young GP, Green TR. The role of single ECG, creatine kinase, and CKMB in diagnosing patients with acute chest pain. Am J Emerg Med 1993;11:444-9.

13 Brush JE, Brand DA, Acampora D, Chalmer B, Wackers FJ. Use of the initial electrocardiogram to predict in hospital complications of acute myocardial infarction. N Engl J Med 1985:312:1137-41.

14 Gibler WB, Runyon JP, Levy RC, Sayre MR, Kacich R, Hattemer CR, et al. A rapid diagnostic protocol and treatment centre for patients with chest pain in the emergency department. Ann Emerg Med 1995;25:1-8.

15 De Leon AC, Farmer CA, King G, Manternach J, Ritter D. Chest pain evaluation unit: a cost-effective approach for ruling out acute myocardial infarction. South Med J 1989;82:1083-9.

16 Zalenski RJ, McCarren M, Roberts R, Rydman RJ, Jovanovic B, Das K, et al. An evaluation of a chest pain diagnostic protocol to exclude acute cardiac ischaemia in the emergency department. Arch Intern Med 1997; 157:1085-91.

17 Tunstall-Pedoe H, Kuulasmaa K, Amouyel P, Arveiler D, Rajakangas A-M, Pajak A. Myocardial infarction and coronary deaths in the World Health Organization MONICA project. Registration procedures, event rates, and case-fatality rates in 38 populations from 21 countries in four continents. Circulation 1994;90:532-612.

18 Sackett DL, Haynes RB, Guvatt GH, Tugwell P. Clinical epidemiology: a basic science for clinical epidemiology, 2nd ed. London: Little, Brown,1991.

19 Ohman EM, Armstrong PW, Christendon RH, Granger CB, Katus HA, Hamm CW, et al. Cardiac troponin T levels for risk stratification in acute myocardial infarction. N Engl J Med 1996;335:1333-41.

20 Hamm CW, Goldmann BU, Heeschen C, Kreymann G, Berger J, Meinertz T. Emergency room triage of patients with acute chest pain by means of rapid testing for cardiac troponin T or troponin I. N Engl J Med 1997;337:1648-53

21 Clements FM, McCann RL, Levin RI. Continuous ST segment analysis for the detection of perioperative myocardial ischaemia. Crit Care Med $1988 ; 16: 710-1$.

22 Krucoff MW, Green CE, Satler LF, Miller FC, Pallas RS, Kent KM, et al. Noninvasive detection of coronary artery patency using continuous ST-segment monitoring. Am J Cardiol 1986;57:916-22.

(Accepted 1 May 2001) 